\title{
Trajectory of Corona Epidemic in India: An Initial Phase Predictive Mathematical Model and the Present Status
}

\author{
Jyoti Bhola ${ }^{1}$, Vandana Revathi Venkateswaran², Monika Koul ${ }^{3}$ and Namrata Dewan Soni ${ }^{4 *}$ \\ ${ }^{1}$ Department of Mathematics, Hansraj College, University of Delhi, Delhi, India. \\ ${ }^{2}$ Research Group for Theoretical Models in Eco-evolutionary dynamics, Max Planck Institute for Evolutionary Biology, Germany. \\ ${ }^{3}$ Department of Botany, Hansraj College, University of Delhi, India. \\ ${ }^{4}$ Department of Physics, Hansraj College, University of Delhi, India.
}

*Corresponding author: Namrata Dewan Soni, Department of Physics, Hansraj College, University of Delhi, India.
Received Date: May 28, 2021

Published Date: July 09, 2021

\begin{abstract}
The novel Coronavirus and its subsequent mutations have been a cause of concern across the world as the human-to-human infection is spreading at a fast pace. Scientists across the world from different disciplines are working to understand the biology, the diseases cycle, epidemiology, and various other attributes of the virus. Mathematicians and statisticians along with computational scientists have come with predictive models that are regional, or state specific. In this paper, we trace a trajectory of the pandemic course in India starting from the initial phase till now, presenting a predictive mathematical model that captured the fate of the virus in the initial phase (till April 2020), indicative data, future projections, and the present status (May 2021) in India. The model predicted that the number of infected people shall increase in India unless physical distancing was implemented in the real sense. The Indian Government with inputs from eminent scientists, laboratory technicians, health care workers, and many excellent predictive models, has done well in containing the pandemic in the first phase. However, owing to the varied environmental conditions prevalent in different areas and mutations in the virus itself, a surge in the number of COVID cases is seen. The model presented in this paper is important as it can provide leads to the health care specialists to prepare the necessary health care facilities and help the rural areas to boost their preparedness to fight the pandemic.
\end{abstract}

\section{Introduction}

Viruses had been considered as inconsequential pathogens in humans for a long time in comparison to plants. The mortality caused by viruses in humans has been exceptionally low in comparison to other diseases such as cancer, cardio-vascular diseases, and tuberculosis (CGHR Report, 2017). However, viruses have been reported to exacerbate the symptoms and have more serious implications on human health if a person is suffering from some auto- immune disorder, infectious disease and has compromised immune system. In India, for diseases such as tuberculosis and cancer, the related mortality rate is so high that researchers and funding agencies have under-estimated the implications that viral diseases can possibly have on public health and socio-economic security. Funding agencies and data scientists have overlooked flu and other human viral pathogens; and in the past years, not much research is funded to work on drug designing or medical research in the field. It is also clear from the recent trends that viral diseases are going to spread at a fast pace, and many novel viruses will be unearthed soon. Climate change and deforestation are also contributing to more frequent outbreaks of viral epidemics. The changing climate is also responsible for such an increase in the 
number of vectors that accelerate the spread of pathogens [1]. In the last few years, WHO (World Health Organization) has been continuously emphasizing that the fast-developing nations, such as India, should triple their expenditure on health care to meet the SDG goals. The outbreak of the SARS CoV- 2 has raised concerns for the Government of India, public policymakers and administration as the pandemic has implications on almost all sectors and strata of the society. It is, therefore, important to understand how the virus will fare in India and how efficiently the country can handle it, without causing severe damage to the human population.

\section{COVID Pandemic}

The recent outbreak of the global pandemic COVID-19 has changed the perspective of everyone in the country regarding viral disease outbreaks as it affects and infects humans in an exponential manner. COVID-19 as well as the causative agent is a novel entry to the viral world and hence has been posing unforeseen challenges. SARS-Cov-2, commonly known as Novel Coronavirus, is a single, positive-stranded, RNA virus belonging to order Nidovirales [2], responsible for the current global pandemic [3]. The virus that first manifested in Wuhan, China has travelled across all seven continents. India, the second most populated country in the world, has been working hard in all dimensions to stop the spread of community infection. Corona viruses show enormous diversity and are evolving fast. Some of the other viruses related to this family of Coronavirus are SARS-CoV, responsible for SARS (Severe Acute Respiratory Syndrome) [4] and MERS-CoV (Middle East Respiratory Syndrome) [5]. Both these viruses emerged from animal reservoirs to cause global epidemics with alarming morbidity and mortality [6]. The reservoir of the Novel Coronavirus is mostly animals found in the wild. Scientists also believe that peri-domestic mammals may also serve as intermediate hosts $[7,8]$. Besides, many spontaneous mutations that keep on happening in nature are facilitating the expansion of genetic diversity [9]. There is strong evidence that human-animal contact at live game markets plays a role and thus, there is the zoonotic transmission of the virus [10].

For long, human viruses have not been considered severe pathogens as infected people develop flulike symptoms and then get cured on their own as the innate immune system triggers antibody formation that provides resistance against the diseases [11-13]. In both developed and developing countries the signs of common flu have not been a cause of concern, though some vaccines have been developed recently and the elderly are advised to take shots yearly as they frequently have a compromised immune system [14]. However, the spread of COVID-19 has alarmed people all over the world. It is important to understand how the virus will fare in an alien environment. Therefore, intensive interdisciplinary research involving biologists, data scientists, mathematicians, clinicians is required to work towards containing the spread of these diseases and design appropriate methods and drugs to mitigate its effects before the situation gets out of hand.
The SARS-Cov-2 virus that first manifested in Wuhan, is different from other Coronaviruses known to humankind. That is the reason why professionals and public alike, have been tremendously cautious of the disease and working consciously to contain the spread. Basic symptoms resemble the normal flulike symptoms that can result in cough, cold, headache and body ache. The more severe consequences include acute respiratory tract infections, that eventually culminate into pneumonia (World Health Organization report, 2020). In more severe cases, (especially among the older population) people develop secondary infections that start affecting other vital organs, and in worst cases, such organ dysfunction(s) lead(s) to the death of individuals who would, otherwise, have recovered from organ failures in absence of the COVID-19 infection [15]. The fast pace at which it spreads from human-to- human contact is the current major reason of worry, and this is what we show through a concise mathematical model in this paper. The infections can spread through the air (if the infected individual is less than one meter apart from uninfected individuals), mostly through the droplets of infected people since the virus stays alive in droplets on the surfaces for many days. As soon as it gets into the host, it replicates in the body and the body becomes a reservoir [16]. However, in some cases, the virus can stay latent inside the body and may not cause any disease symptoms. But if this person is a carrier, then he/she can spread the disease to others who come in contact with the droplets (that are released during coughing or sneezing) of this person. The symptoms resemble people who catch seasonal flu-like illness, ranging from cough, cold, fever to shortness of breath $[17,18]$. As the symptoms shown by the person infected with novel Coronavirus are similar and overlap with common flu, it is not easy to identify the carriers and therefore, the transmission cannot be easily contained. Recent studies have indicated at involvement of the hemostatic system in severe COVID-19 pneumonia and endothelial dysfunction which if untreated affects other biological processes [19].

First few reported cases of COVID-19 infected people had shared history of human-animal contact at live game markets. The World Health Organization has declared it a pandemic after more than 200,500 confirmed cases of infection and more than 9,000 deaths across 115 countries (World Health Organization, 2020). The virus epidemiology has been understood in China where research teams, institutes and scientists, and various cross sectors worked in coordination to control the infection spread. Today China has successfully been able to control it in the worst affected areas $[20,21]$. This shows the importance of coordinated and well-funded research, especially in fields crucial to the survival of humankind. India tops the list of countries with high population and has been trying hard to negotiate the problems and challenges thrown by this overwhelming population (Ministry of Health, GOI, 2020). The rise in population has already created a crisis for the health care sector and the facilities for primary and secondary education. With the outbreak of the novel Coronavirus, the country must gear up to 
confront it. It is important for Indian scientists to come together and study pathogenicity in the Indian context.

In India, the first case was reported at the end of January 2020, and the number had grown to touch 800 by March 28, 2020. But India is a peninsula, and the temperature, humidity, and topography are variable, so the factoring of all these variables also has an implication on mortality and morbidity. Also, education, awareness and understanding of people, socio- economic status is variable, so the infection percentage and the magnitude of impact are also different. This calls for regional data assessment and modeling. Besides, mathematical, and ecological modeling can help in predicting the disease course. The data generator through these models can consider various variables that are specific to the country and give some predictions. This can help in a fair assessment and put all the fake and unscientific assumptions on hold. This data can also help in giving recommendations to the health care agencies. Tiwari (2020) [22] used SIR and modified SIQR modelling to approach and analyze the impact of COVID on some countries using certain assumptions. Aquas et al (2020) [23] used mathematical modelling to provide evidence for pandemic containment strategies. According to these studies, high-income Asian countries were able to call on their system responsiveness by rapidly enforcing efficient testing and quarantining/isolation strategies. Other low-income countries, and even some regions within India have successfully enforced such efficient systems.

Ranjan et al (2021) studied the characteristics of COVID-19 spread for some Indian states by estimating test positivity and case fatality rates. It has been pointed out the virus is behaving in an erratic manner and the symptoms are highly diverse, and that is a cause of concern for medical practitioners. The present study has proven to be valid and the surge in cases followed the trend in tune with our analysis. Hence, physical distancing, wearing of masks, and government guidelines should be followed. The emergence of yet another outbreak of human disease, caused by a pathogen from a viral family, formerly thought to be relatively benign, underscores the perpetual challenge of emerging infectious diseases and the importance of sustained preparedness. Major gaps in our knowledge of the origin, epidemiology, duration of human transmission, virus evolution and clinical spectrum of the disease need fulfillment by future studies.

Research teams including biologists, biotechnologists, and medical practitioners all over the world are trying to understand biology, etiology, and epidemiology of the diseases (Epidemiology working group, 2020; [16,24]). Mathematicians, data scientists and statisticians have proposed various models to decipher and predict the behavior of the epidemic in various environments [2528]. Scientists have also been using dynamic bipartite graphs to model the physical contact patterns that result from movements of individuals between specific locations based on the trends available each day $[29,30]$.

\section{Model}

Initial phase in India: We used a Computer Algebra System, Mathematica, to do predictive modeling, based on the data available on mortality due to the pandemic in the initial phase (till April 2020). In this context, the population of India can be classified into three broad classes, namely:

$I(t):$ the number of infectives

$\mathrm{S}(\mathrm{t})$ : the number of susceptible

$\mathrm{R}(\mathrm{t})$ : the number recovered (recovered, died, or naturally immune to the disease)

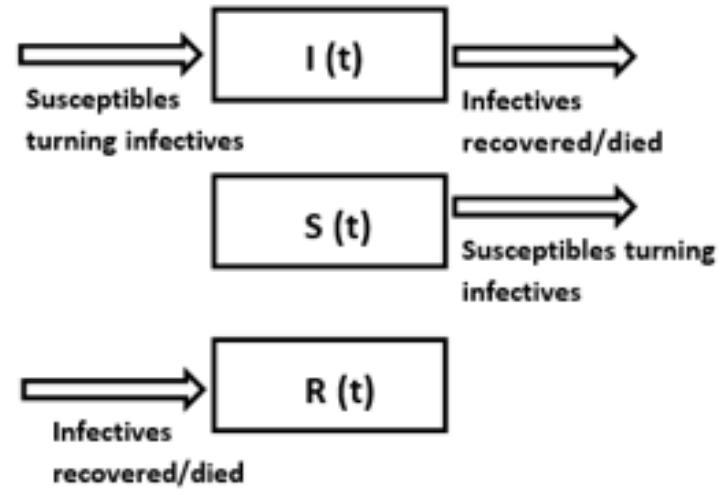

Not considering virus evolution

Susceptibles turning infectives Infectives turning into dead or recovered

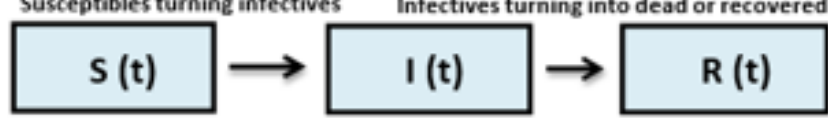

Considering virus evolution

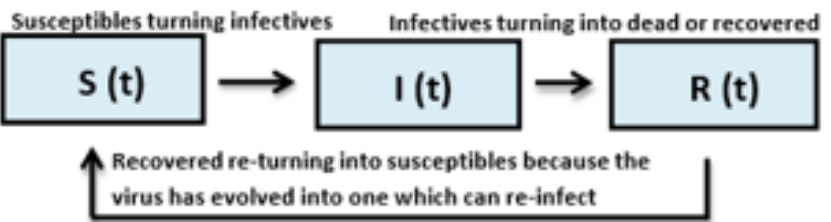

Figure 1: Compartmental diagram. A flowchart of the SIR (Susceptible-Infected-Recovered) process.

Clearly, $\mathrm{I}(\mathrm{t})+\mathrm{S}(\mathrm{t})+\mathrm{R}(\mathrm{t})=\mathrm{N}$ where $\mathrm{N}$ is the total population of India ( $\mathrm{N}$ is itself a variable, but treated as a constant in this model, consistent with the fact that the course of the epidemic is short compared to the lifetime of an individual). This can be seen as a three-compartment model (Figure 1), and it is in our interest to find the minima of the $\mathrm{I}(\mathrm{t})$ compartment under the prevailing 
situations. A minima of $\mathrm{I}(\mathrm{t})$ compartment would mean that the number of infected people is at its lowest. Decreasing $I(t)$ is what is in the interest of the state and scientists. From what is known about Coronaviruses, it is evident that the per capita rate of increase in the number of infectives is directly proportional to the number of susceptible in the vicinity of an infective and hence, the total intake in the first compartment looks like (kS)I; where $\mathrm{k}$ signifies the rate of transmission indicated by the average number of people who will catch the virus from one infected person.

The differential equation system for the basic SusceptibleInfected-Recovered or SIR Model (Barnes and Fulford, 2002) is given by,

$$
\frac{d I}{d t}=k S I-r I
$$

$$
\begin{aligned}
& \frac{d S}{d t}=-k S I \\
& \frac{d R}{d t}=r I
\end{aligned}
$$

where $r$ is the rate at which infectives recover or die; and clearly, these individuals can no longer remain infective. Looking at the initial statistics worldwide (up to April of 2020), the value of $\mathrm{k}$ was somewhere between 1.4 and 2.5 (World Health Organization, 2020) [17]. For the sake of visualization, let us take the total world population to be 1000 and a single infective to begin with. A plot code in Mathematica for $\mathrm{k}=2$ gives a striking sketch (Figure 2) as under: Starting with just a single infective, the infection peaks up to almost the total population size before starting to decrease. The initial phase situation is India is summarized in (Figure 3).

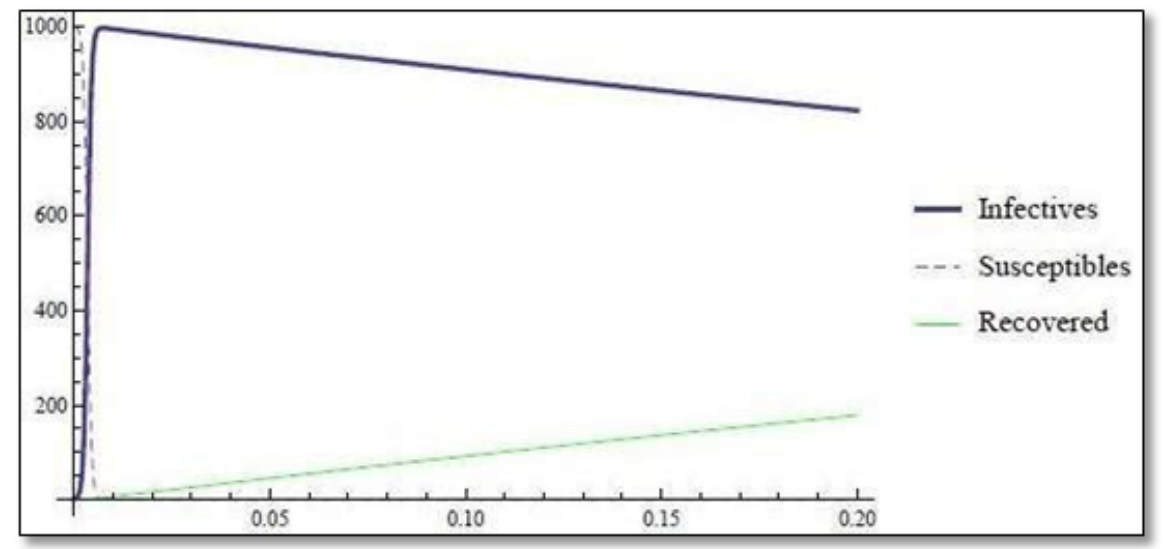

Figure 2: Model plot for $\mathrm{k}=2$.

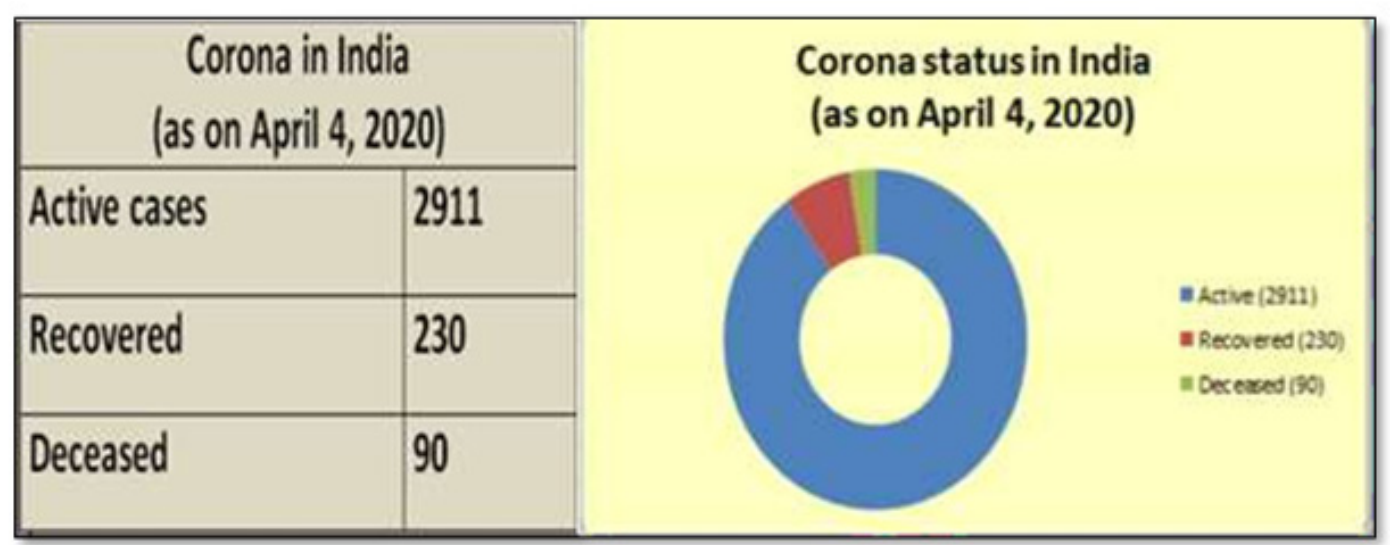

Figure 3: Initial status in India.

Until April 2020, community transmission was not validated, and the $\mathrm{k}$ value was significantly less than 1 in India. The infection was still projected to continue growing before attaining a peak lower than the total susceptible population, as indicated by the following plot (Figure 4). To contain the transmission of this virus, it is extremely important to contain the value of $\mathrm{k}$. This $\mathrm{k}$ value is supposedly dependent on many factors that involve both natural (temperature, humidity) and non-natural factors or personal 
provisioning measures (physical distancing, infective wearing masks, good hygiene practices such as washing hands with soap for 20 seconds, and so on). Also, from the above plots (Figures 2 $\& 4$ ), though the transmission rate was very low in India initially, the number of infectives was bound to increase with time. The only way to get the graph of infectives as a decreasing function of time as per the proposed model is that the interaction term kSI in equation (1) tends to zero. That was possible only when infective were totally isolated from the susceptible population. The variables kept on changing with each passing day as the virus had just reached stage 2 of its disease cycle where there were no mass casualties and things were under control [31,32].

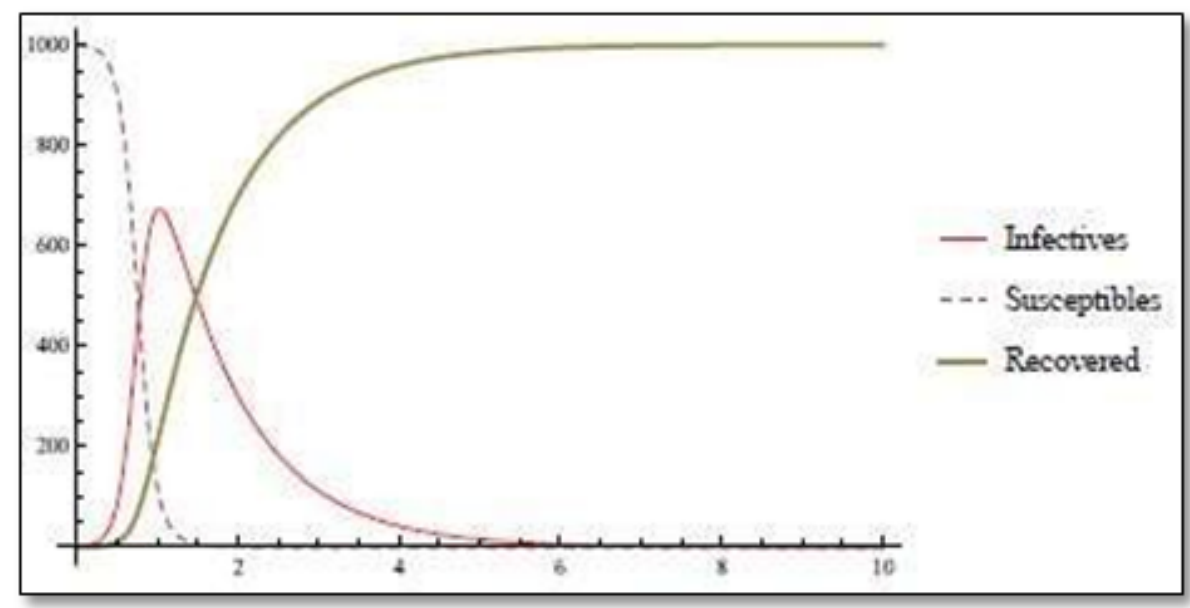

Figure 4: Model plot for initial phase (for $\mathrm{k}=0.01$ ).

The k- value being greater than unity implies exponential growth of the disease as every infected individual replaces himself or herself with more than one new infected person on an average. Scientists classify this value as R0 or basic reproduction number. The R0 for measles is around 12, the R0 for COVID-19 is around 2.6, and for seasonal flu it is around 1.3. Figure 5 shows how the number of new cases (per transmission) for seasonal flu is negligible as compared to COVID-19. The state wise scenario in India as on April 4, 2020 is charted in (Figure 6). (Data Source: https://www.covid19india.org/). The chart in Figure 6 shows the maximum number of COVID-19 cases in Maharashtra. Figure 7 depicts a positive correlation between the population density per square mile and number of cases for 7 states topping the list in COVID-19 chart shown in (Figure 6).

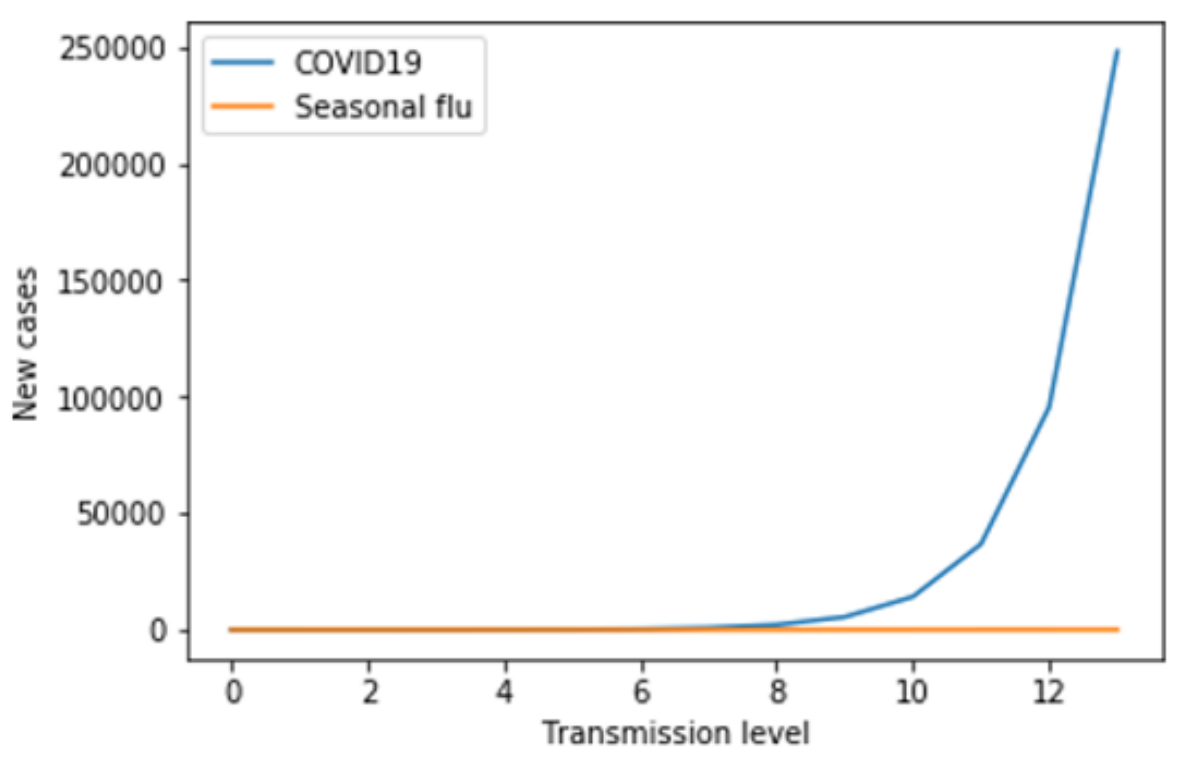

Figure 5: Transmission of COVID 19 vs. Seasonal Flu. 


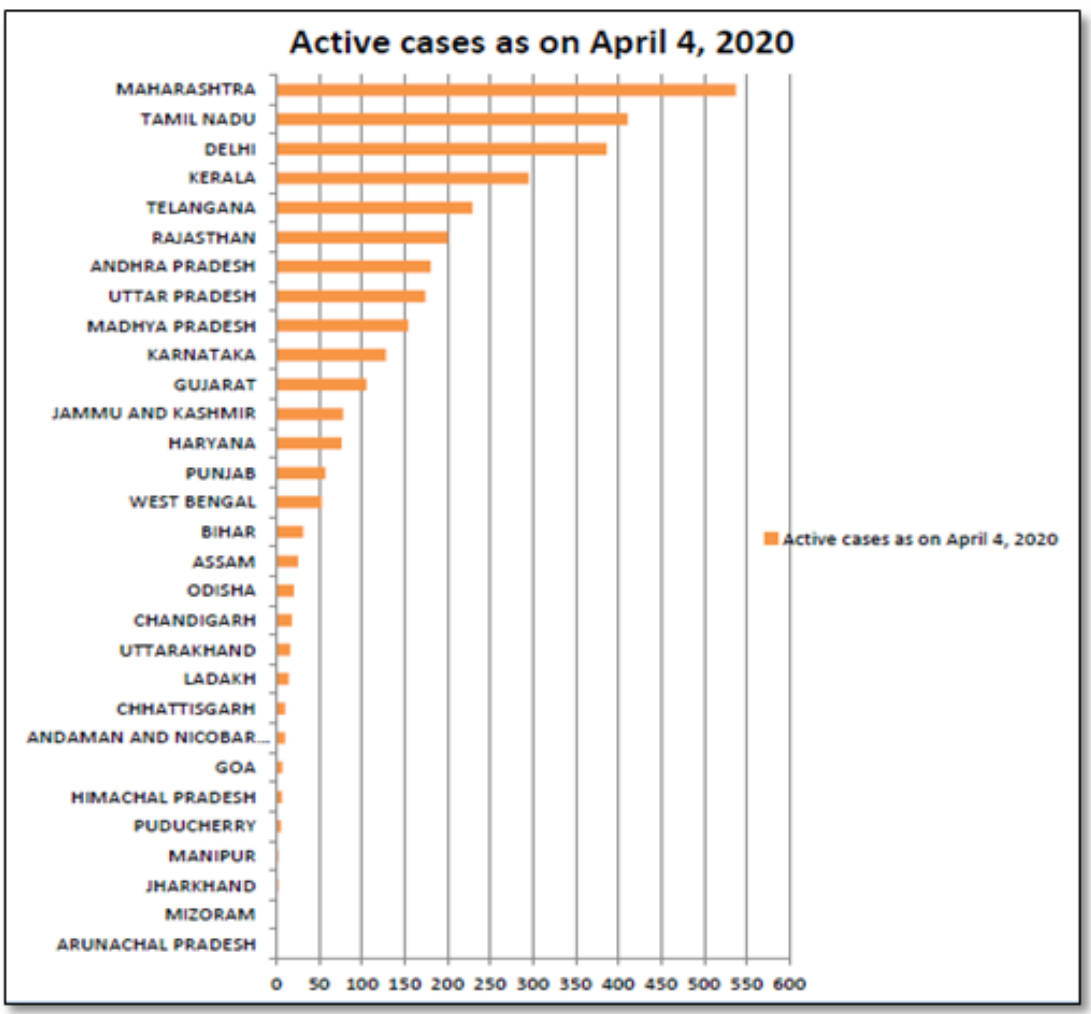

Figure 6: State-wise active cases in India (as in April 2020).

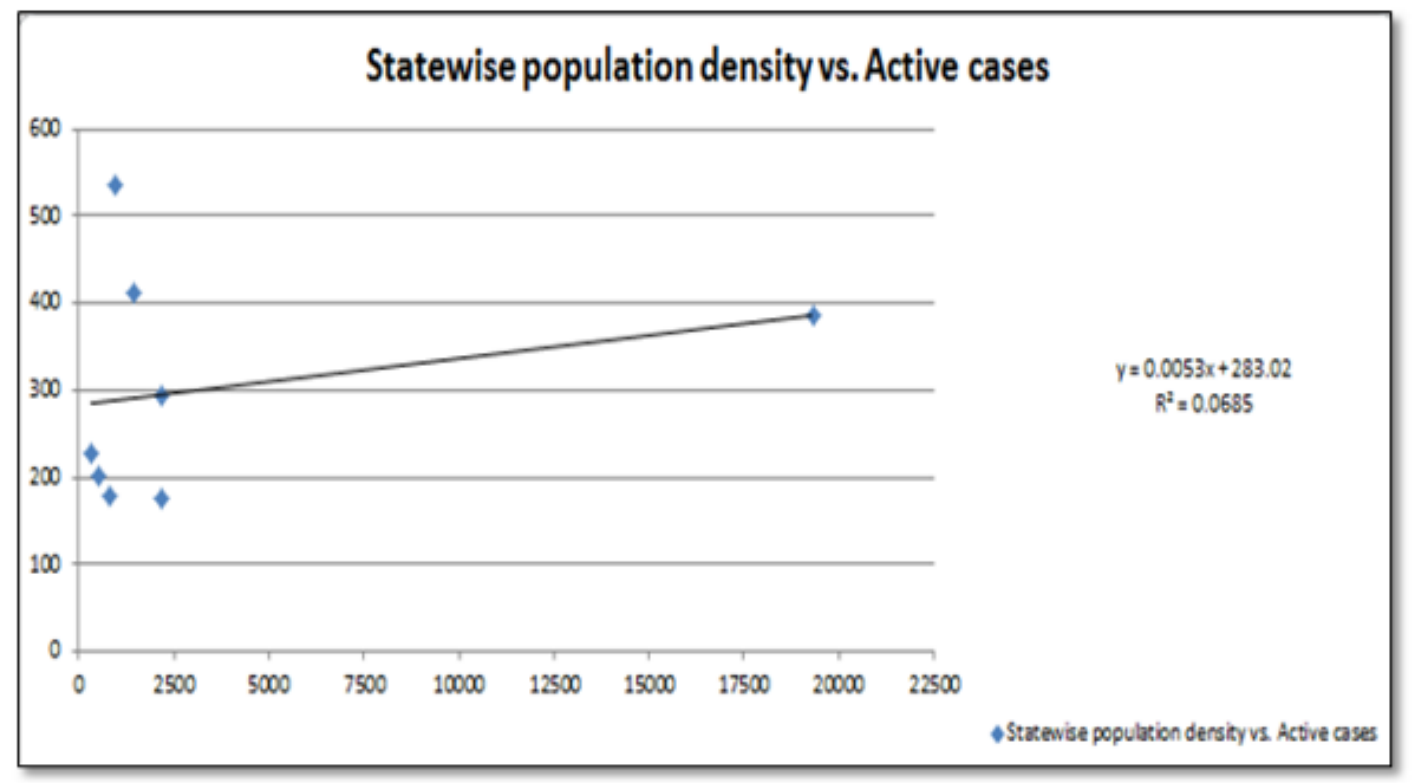

Figure 7: Correlation between population density and Active cases (as in April 2020).

Maharashtra is densely populated with Mumbai being an overcrowded city that has a population size of more than 20 million people to which this highest number is being attributed to. This has happened in Maharashtra even when the state has not been the first one to get the infection in India. The same can be paralleled with the Influenza epidemic a century ago which took a toll of estimated 10-20 million people in India alone and warned us of what lied ahead of us, if the initial situation summarized above went uncontrolled, unattended, or casually attended. The numbers kept on fluctuating for months with phases of sealing and unlocking 
all over, people taking COVID appropriate protocols and workplaces going functional in phases. The panic had subsided to quite an extent when there was the second wave knock at the door.

\section{Present Status in India}

India is presently facing the second wave of infection, which was apprehended much earlier by scientists working on the evolution of this new virus. The new mutant in India is far more infectious than its predecessor and the infection is spreading like a wildfire in all the states. Though the recovery rate is still high in India, the panic all around is a cause of worry and disturbance nationwide. The statistics at present are illustrated in (Figures 8 and 9) below. Maharashtra is still topping the infection chart as in the initial phase, most likely owing to high population density, as pointed out in the analysis of the initial phase [33,34]. Right from the onset of the pandemic, health care facilities are being updated; medical and paramedical staff are getting trained, and many agencies are raising awareness on the issues related to this virus and its transmission.

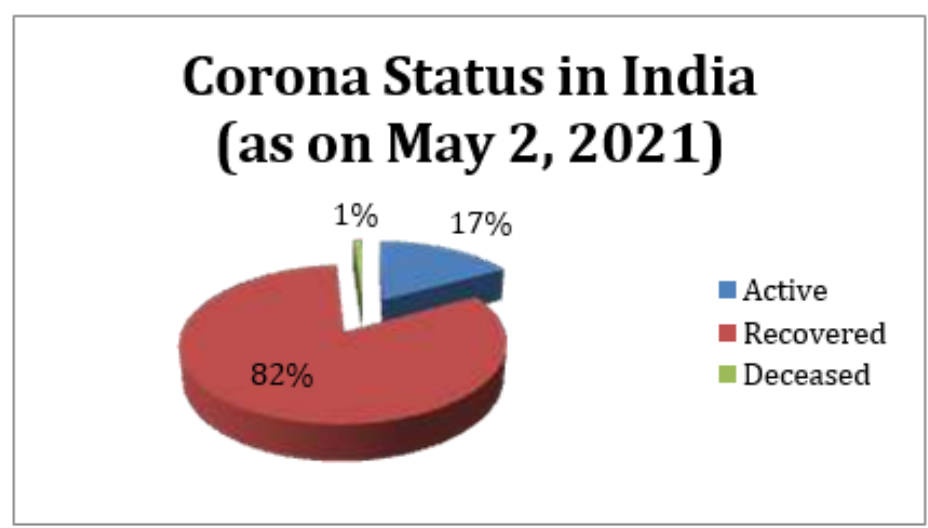

Figure 8: Present status in India (as in May 2021).

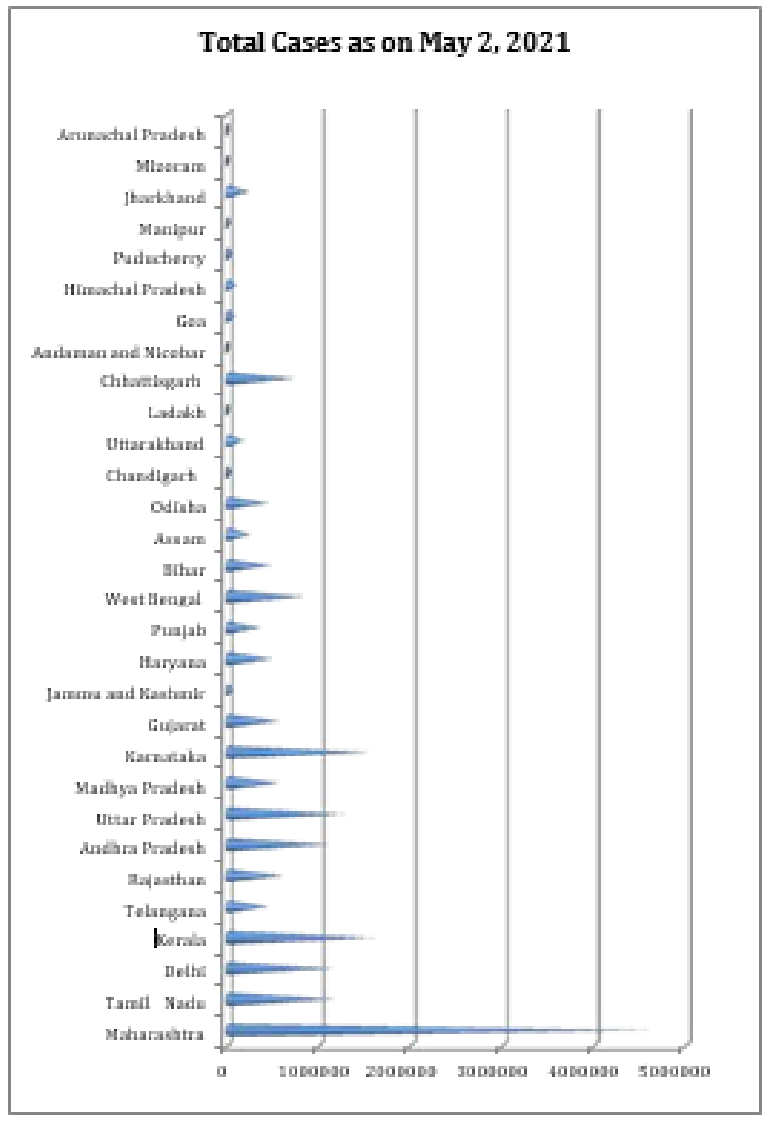

Figure 9: State-wise total COVID cases in India (as of May 2, 2021). 
Different states have adopted measures to stop the community spread but the new mutants popping up has made it difficult to predict the spread of the virus and its fate in the Indian context. Considering the vast size of the country, the population size, and other socio-economic conditions of the states, a single uniform policy cannot work to contain the disease. The vaccine is available now and the vaccination drive is going on in phases (as per the age groups, healthcare workers, etc). However, it is still a challenge to get every person in the country vaccinated as the population is so huge. Besides, false notions and negative campaigning against vaccination are going on in various parts and some communities are reluctant to get vaccinated.

\section{Conclusions and Recommendations}

Data analysis over a span of one year suggests that the country is seeing a surge in the cases. However, variables such as hygiene, physical distancing, staying indoors, and boosting the immune system can flatten the curve, and when these were followed, they did flatten the curve. Raising awareness through various platforms including the social networking websites like Facebook and Twitter is one way of containing the disease, and we must keep doing it. Each household must be reached through vernacular language since a large section of the population is still not conversant in English and Hindi. The Government has taken measures such as setting testing centers and has designated isolation blocks in hospitals. This would ease the burden on the existing ones. New makeshift health centers can also help as they did in China, which helped them win over the battle despite the sudden high cases there.

Also, the lockdown in the country announced by the Government surely contained the spread of infection and helped India attend to this new challenge in the desired form. Strict measures have been announced for people who are not following Government Advisory. It is important to boost the morale of front-line workers (such as medical practitioners and paramedical staff, nursing staff, cleaning staff, and housekeeping departments of the hospitals and health care centres) who are interacting directly with the patients. People who are showing anxiety symptoms due to restrictions and lockdowns need constant counseling. Psychologists, self-help groups need to offer counseling through electronic media. Indians also need to fight the menace of superstitions and myths that are being propagated regarding the cure of Corona-related illness. Scientists have also geared up for the task and come forward to do collaborative research work to understand the spread, containment, and eventualities of the pandemic outbreak. For this, interdisciplinary teams have been working together to come out with some concrete strategies. Research teams must develop vaccines for which funding, infrastructure, and adequate facilities are required, now and for future possible issues.

Strategies for addressing respiratory failure is the need of hour. Protective mechanical ventilation and high- flow nasal oxygen (HFNO) or non-invasive ventilation (NIV) facilities are required in all hospitals and COVID centres. COVID-19 is a reminder that science cannot take a back seat, and health care, education, and research should always hold a top priority. Phase wise vaccination drive is going on in the full spree in India. Vaccines were being given to the elderly and those showing comorbidity at first, then to people above 45 years of age and India had announced vaccine administration to all above 18 years of age from May 1, 2021 onwards. However, the population of the country is huge, and we still need research on vaccines so that every person receives the shot. Also, there are chances of other microbes and pathogens spreading as well. So, 'preparation and mitigation' is the way forward. Faith in science and scientists, and optimism are important at this juncture so that India comes out as a winner in the battle.

\section{Data Accessibility Statement}

There is no data to be archived. The Mathematica code that we used can be provided upon request to the author of correspondence.

\section{Acknowledgement}

None.

\section{Conflict of Interest}

The authors declare no conflict of interest.

\section{References}

1. Khan MD, Hong Ha Thi Vu, Quang Tuan Lai, Ji Whan Ahn (2019) Aggravation of Human Diseases and Climate Change Nexus. Int J Environ Res Public Health 16(15): 2799

2. Cascella M, Rajnik M, Cuomo A, Dulebohn SC, Di Napoli R (2020) Features, Evaluation and Treatment Coronavirus (COVID-19). In StatPearls [Internet]. StatPearls Publishing.

3. Huang C, Yeming Wang, Xingwang Li, Lili Ren, Jianping Zhao, et al. (2020) Clinical features of patients infected with 2019 novel coronavirus in Wuhan, China. The Lancet 395(10223): 497-506.

4. Thomas G Ksiazek, Dean Erdman, Cynthia S Goldsmith, Sherif R Zaki, Teresa Peret, et al. (2003) A novel coronavirus associated with severe acute respiratory syndrome. N Engl J Med 348(20): 1953-1966.

5. Noah C Peeri, Nistha Shrestha, Md Siddikur Rahman, Rafdzah Zaki, Zhengqi Tan, et al. (2020) The SARS, MERS and novel coronavirus (COVID-19) epidemics, the newest and biggest global health threats: what lessons have we learned?. Int J Epidemiol 49(3): 717-726.

6. Cavanagh D (2007) Coronavirus avian infectious bronchitis virus. Vet Res 38(2): 281-297.

7. Malik YS, et al. (2020) Emerging Coronavirus Disease (COVID-19), a pandemic public health emergency with animal linkages: Current status update. Preprints, doi: https://doi.org/10.20944/ preprints202003.0343.v1.

8. Murdoch DR, French NP (2020) COVID-19: another infectious disease emerging at theanimal-human interface. NZ Med J 133(1510): 12-15

9. He X, Yue W, Yan J (2020) SNV Reoccurrence in Multiple Regions in the Genome of 2019-nCoV. Preprints, 2020020132.

10. Li Q M Med, Xuhua Guan, Peng Wu, Xiaoye Wang, et al. (2020) Early transmission dynamics in Wuhan, China, of novel coronavirusinfected pneumonia. New England Journal of Medicine 382: 1199-1207.

11. Chiu CY (2013) Viral pathogen discovery. Curr Opin Microbiol 16(4): 468-478. 
12. Amy Kistler, Pedro C Avila, Silvi Rouskin, David Wang, Theresa Ward, et al. (2007) Pan-viral screening of tract infections in adults with and without asthma reveals unexpected human coronavirus and human rhinovirus diversity. J Infect Dis 196(6): 817-825.

13. Wrammert J, Kenneth Smith, Joe Miller, William A Langley, Kenneth Kokko, et al. (2008) Rapid cloning of high-affinity human monoclonal antibodies against influenza virus. Nature 453: 667-671.

14. Voordouw ACG, MCJM Sturkenboom, JP Dieleman, Th Stijnen, D J Smith, et al. (2004) Annual revaccination against influenza and mortality risk in community-dwelling elderly persons 292(17): 2089-2095.

15. Xu Z, Lei Shi, Yijin Wang, Jiyuan Zhang, Lei Huang, et al. (2020) Pathological findings of COVID-19 associated with acute respiratory distress syndrome. The Lancet respiratory medicine 8(4): 420-422.

16. Rothan HA, Byrareddy SN (2020) The epidemiology and pathogenesis of coronavirus disease (COVID-19) outbreak. J Autoimmun 109: 102433.

17. World Health Organization (2020) Report of the WHO-China Joint Mission on Coronavirus Disease 2019 (COVID-19).

18. Wu Z, McGoogan JM (2020) Characteristics of and important lessons from the coronavirus disease 2019 (COVID-19) outbreak in China: summary of a report of 72314 cases from the Chinese Center for Disease Control and Prevention. JAMA 323(13): 1239-1242.

19. Balasubramanian Ganesh, Thangarasu Rajakumar, Mathiyazhakan Malathi, Natesan Manikandan, Jaganathasamy Nagaraj, et al. (2021) Epidemiology and pathobiology of SARS-CoV-2 (COVID-19) in comparison with SARS, MERS: An updated overview of current knowledge and future perspectives. Clin Epidemiol Glob Health 10 100694.

20. coronavirus diseases (COVID-19) in China. Epidemiology Working Group for NCIP Epidemic Response, Chinese Center for Disease Control and Prevention 41(2): 145-151.

21. Zhang Rongqiang, et al. (2020) Transmission and epidemiologica characteristics of severe acute respiratory syndrome coronavirus 2 (SARS-CoV-2) infected pneumonia (COVID-19): Preliminarily evidence obtained in comparison with 2003-SARS. MedRxiv.

22. Tiwari A (2020) Modelling and analysis of COVID-19 epidemic in India. Journal of Safety Science and Resilience 1(2): 135-140.
23. Aguas R, White L, Hupert N, Rima Shretta, Wirichada Pan Ngum, et al. (2020) Modelling the COVID-19 pandemic in context: an international participatory approach. BMJ Glob Health 5: e003126.

24. Lipsitch M, Swerdlow DL, Finelli L (2020) Defining the epidemiology of Covid-19—studies needed. N Engl J Med 382(13): 1194-1196.

25. Sandip Mandal, Tarun Bhatnagar, Nimalan Arinaminpathy, Anup Agarwal, Amartya Chowdhury, et al. (2020) Prudent public health intervention strategies to control the coronavirus disease 2019 transmission in India: A mathematical model-based approach. Indian J Med Res 151(2): 190199.

26. Nesteruk I (2020) Statistics based predictions of coronavirus 2019nCoV spreading inmainland China. MedRxiv.

27. Ming WK, Huang J, Zhang CJ (2020) Breaking down of healthcare system: Mathematical modelling for controlling the novel coronavirus (2019-nCoV) outbreak in Wuhan, China.

28. Maier BF, Brockmann D (2020) Effective containment explains subexponential growth in confirmed cases of recent COVID-19 outbreak in Mainland China. arXiv preprint arXiv:2002.07572.

29. Zhao S, et al. (2020) Estimating the serial interval of the novel coronavirus disease (COVID-19):a statistical analysis using the Public data in Hong Kong from January 16 to February 15, 2020. medRxiv.

30. Barnes B, Fulford GR (2015) Mathematical Modelling with case studies, A differential equation approach using Maple and MATLAB. Third Edition CRC Press, Taylor and Francis, London and NewYork.

31. Eubank S, Guclu H, Kumar VA, Marathe MV, Srinivasan A, et al. (2004) Modelling disease outbreaks in realistic urban social networks. Nature 429(6988):180- 184

32. Adam J Kucharski, Timothy W Russell, Charlie Diamond, Yang Liu, John Edmunds, et al. (2020) Early dynamics of transmission and control of COVID-19: amathematical modelling study. Lancet Infect Dis 20(5): 553558.

33. Perlman S, Netland J (2009) Coronaviruses post-SARS: update on replication and pathogenesis. Nat Rev Microbiol 7(6): 439-450.

34. Yang Yang, Qing-Bin Lu, Ming-Jin Liu, Yi-Xing Wang, An-Ran Zhang, et al. (2020) Epidemiological and clinical features of the 2019 novel coronavirus outbreak in China. 